\title{
Research on DISO DC-DC Converter Based on the Topology of Push-Pull M2DC
}

\author{
Wei Lin ${ }^{1, a}$, Yihui Zheng ${ }^{1, b^{*}}$, Lixue $\mathrm{Li}^{1}$, Xin Wang ${ }^{1}$, Chunming Zhao ${ }^{2}$ and \\ Jianping Lie ${ }^{2}$ \\ ${ }^{1}$ Center of Electrical \& Electronic Technology, Shanghai Jiao Tong University, Shanghai, China \\ ${ }^{2}$ State Grid Jilin Province Electric Power Research Institute, Changchun, China \\ asleepsleeper@163.com, bzhengyihui@sjtu.edu.cn \\ corresponding author: zhengyihui@sjtu.edu.cn
}

Keywords: DC-DC converter, Modular, Power loop, DISO

Abstract. In order to improve the flexibility and make energy transmissing between power system and distributed generators more efficient, a double-input single-output DC-DC converter based on the topology of push-pull modular multilevel DC converter is presented in this paper. First, it selects the topology structure of which converter can work in high voltage field and avoid inconvenience and hidden dangers which exist in AC connection. Second, aiming at the defect of general DC system, easy to be affected by AC system, the coupled inductor is used to build the topology similar to boost converter and add the filter function which can prevent $\mathrm{AC}$ component into input and output side to ensure DC side stabilization. Then, the voltage source and a coupled inductor are added into low voltage side to guarantee the flow type of current in the secondary power loop which can make independence between two input sources. The independence makes it possible to control the input sources separately, which ensures the reliability of output power and increases flexibility of input sources. Finally, from the simulation results, it is concluded that the topology is feasible.

\section{Introduction}

In recent years, a variety of issues such as greenhouse effect, growth of energy demand, and wastage of energy catch people's eyes. A number of Distributed Generations (DGs) are installed into the power system to reduce the environmental and energy problems. As the result, microgrids are especially researched all over the world[1]. On the other hand, High-Voltage Direct Current (HVDC) transmission has become a mature technology to efficiently transfer large amounts of energy across far distance in the past years[2]. It needs more useful DC-DC converters to control these power flow in the grids so that the system can run efficiently more[3].

In the application scenario for high-voltage and medium-voltage, Modular Multilevel Converter (M2C) has advantage comparing with another topologies. In 2002, Marquardt and Lesnicar came up with that $\mathrm{M} 2 \mathrm{C}$ was a $\mathrm{AC}-\mathrm{DC}$ converter topology with great prospect between high-voltage power systems[2]. And high-voltage DC-DC converter can be gotten by making two M2C equipments connect back-to-back with each other. Efficiency of the structure found by Kenzelmann will be over 99\%[4]. Basing on the background, Modular Multilevel DC Converter (M2DC) inherited many advantages of $\mathrm{M} 2 \mathrm{C}$, such as economical efficiency, conveniences. What's more, the superiority of M2DC was that it changed voltage level by DC-DC. They will make M2DC play a important character in the power systems in the future. And push-pull M2DC was one topology with good development prospect. However, push-pull M2DC was Single-Input Single-Output (SISO) DC-DC converter. It was exposed some disadvantage for developing microgrids and sorts of applications for high-voltage and medium-voltage power system.

In this paper, a Double-Input Single-Output (DISO) DC-DC converter based on the topology of push-pull M2DC is presented. After a comparison, the push-pull M2DC is selected. It can work in high voltage field and avert hidden trouble which appears in AC connection. Then, adding some elements such as coupled inductors and voltage source to solve the problem met: the coupled inductors are 
installed to filter AC component and ensure the independence between input sources, and the voltage source added should play the character of the other input source. The DISO push-pull M2DC makes it possible to allot power flow by controlling the two input sources separately. And this topology built with the independence of input sources will get higher flexibility and better stability for power system. It is feasible as shown in running result of topology by simulating. It should make the energy transmissing of poewr system and DGs become more efficiency in the future.

\section{Theory and Structure Analysis of Push-Pull M2DC}

According to Fourier series analysis, non-sinusoidal voltage and current can be expressed by the sum of different frequencies sinusoidal functions. Because of the orthogonality of sinusoidal function, the decoupling of active power at different frequencies creates the possibility that a converter can generate an active power at one frequency by absorbing the same active power at another frequency and it needn't get power from additional power source.

The submodules (SM) with capacitor converting power between frequencies have three possible changes: half-bridge, full-bridge and full-bridge with a transformer. Many of power cells or submodules are connected in series and controlled to convert power between two different frequencies. DC, or zero frequency, is a valid frequency, too.

Push-pull M2DC comprises four bridges and two coupled inductors. They control output voltage by commanding the number of submodules and duty cycle of switches to get different input-output ratio.From the power equilibrium theory, the secondary power loop achieves power converted by using $\mathrm{AC}$ frequency which is different from zero frequency (DC). And AC component will not influence input voltage source through the coupled inductor $L_{\text {high }}$ and $L_{\text {low }}$.

\section{Research of DISO Push-Pull M2DC}

There is a circuit added which includes a bottom shunt submodule and low voltage source in parallel in the DISO push-pull M2DC comparing with the normal push-pull M2DC. Shown in Fig. 1.

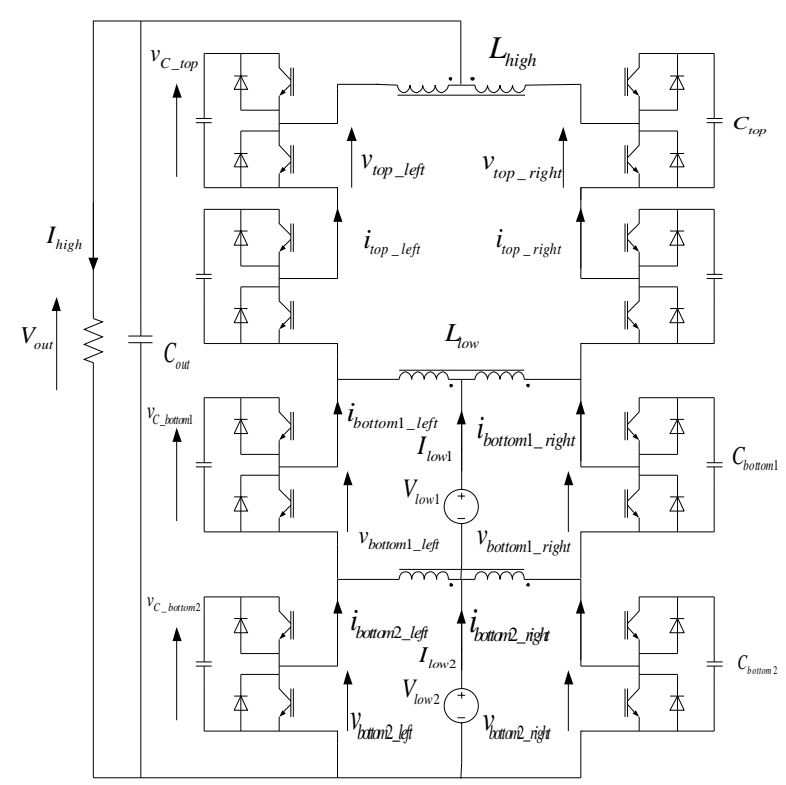

Fig. 1. Topology of DISO push-pull M2DC

Since output voltage of bottom submodule is decided on the input voltage $V_{\text {low } 1}$ and $V_{\text {low } 2}$ in parallel only, it should not influence the running of original bottom submodule to increase or decrease the number of another input voltage sources.

As DISO push-pull M2DC, upper and lower bottom shunt submodules are controlled by input source. And top series submodule can control the output voltage on request. On the other hand, input 
voltage mustn't be low voltage level in DISO push-pull M2DC, it can be used that a low voltage side and high voltage side as input together, and the other low voltage side as output.

\section{Control of Push-Pull M2DC}

For this circuit, whatever in primary power loop or secondary one, it must be controlled by turn-off or turn-on of switch finally. It is most important to control the voltage of capacitors to equalize by secondary power loop for bottom submodules.
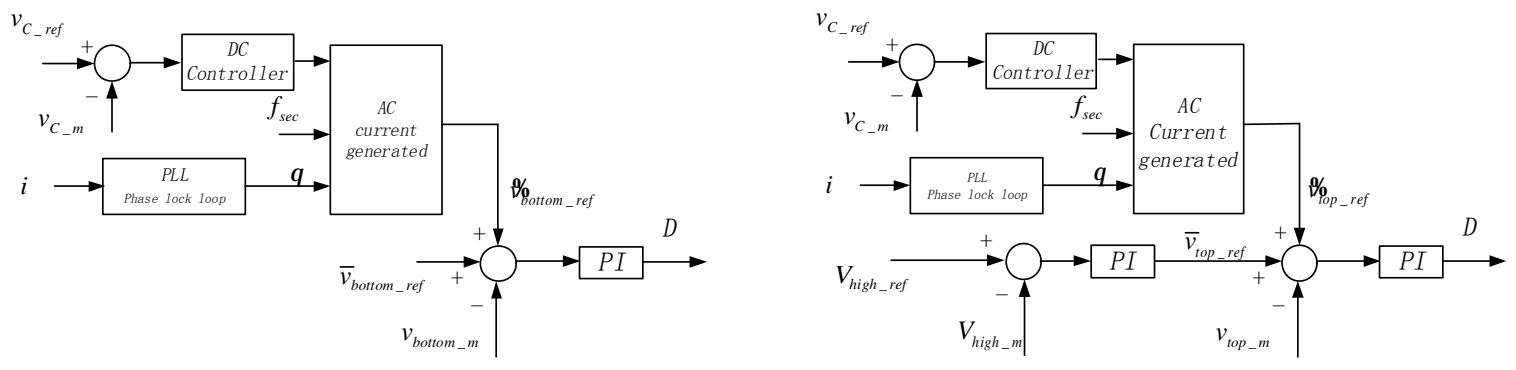

Fig. 2. Control block diagram of bottom shunt and top series submodules of M2DC

In Fig. 2, where $v_{C_{-} \text {ref }}$ is the referential voltage of capacitor in the bottom shunt submodule, and $v_{C_{-} m}$ is the measure value of capacitor voltage in fact. It is generated by DC controller which is the amplitude of $A C$ voltage reference of bottom shunt submodule. For top series submodules, besides the voltage balanced which is similar to bottom submodules, it should be attended to control the output voltage of itself. They are shown in Fig. 2.

\section{Simulation of Push-Pull M2DC}

For the sake to simplify, this paper uses the step-up ratio 3, the number of bridge of top series submodule and bottom shunt submodule are 4 and 2 . And the parameters used are shown in Table 1.

Table 1. Simulation parameters of push-pull M2DC

\begin{tabular}{c|c}
\hline Parameter & Value \\
\hline Input voltage & $380[\mathrm{~V}](\mathrm{DC})$ \\
Output voltage & $2.5[\mathrm{kV}](\mathrm{DC})$ \\
Capacitor voltage of top SM & $1.3[\mathrm{kV}](\mathrm{DC})$ \\
Capacitor of top SM & $200[\mu \mathrm{FF}]$ \\
Capacitor of bottom SM & $60[\mu \mathrm{F}]$ \\
Load resistance & $312.5[\Omega]$ \\
Output side coupled inductor & $10[\mathrm{mH}](30 \%)$ \\
Switch frequency & $1[\mathrm{kHz}]$ \\
Secondary power loop frequency & $60[\mathrm{~Hz}]$ \\
Output voltage of bottom SM (DC) & $380[\mathrm{~V}](\mathrm{DC})$ \\
Output voltage of bottom SM (AC) & $20[\mathrm{~V}](\mathrm{AC})$ \\
Input side coupled inductor & $5[\mathrm{mH}](20 \%)$ \\
Capacitor voltage of bottom SM & $1.25[\mathrm{kV}](\mathrm{DC})$ \\
\hline
\end{tabular}

It is shown in Fig. 3, Fig. 4 and Fig. 5 that the voltages are satisfied mainly. For DISO push-pull M2DC, the voltage of output equals to the sum of two input sources. The voltage shown in Fig. 4 expresses the same result. So the topology of this paper is feasible. However, the waveform in these figures are not perfect that should be improved in the future. 

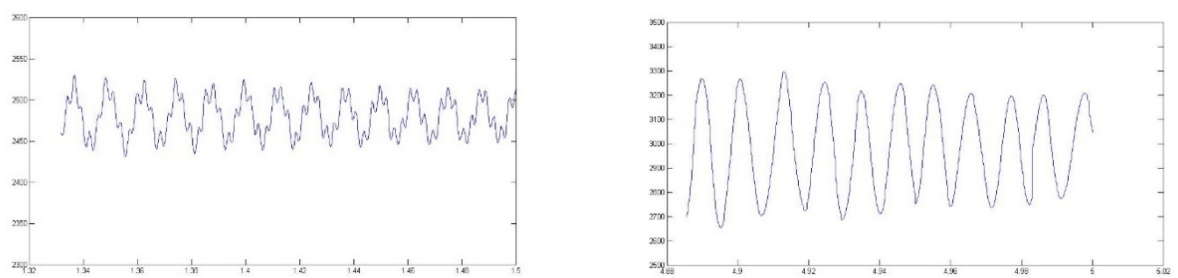

Fig. 3. Output voltage of push-pull M2DC and DISO push-pull M2DC
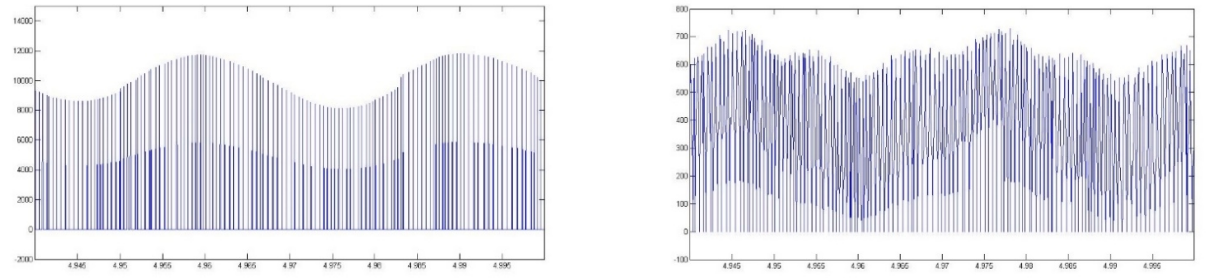

Fig. 4. Voltage of input source 1 and input source 2 of DISO push-pull M2DC
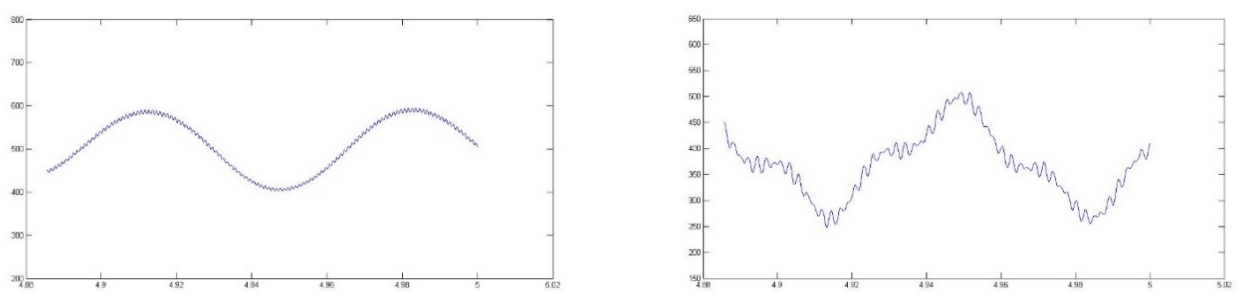

Fig. 5. Filtering voltage of input 1 and input 2 of DISO push-pull M2DC

\section{Conclusions}

A DISO DC-DC converter based on the topology of push-pull M2DC is proposed in this paper. For allotting the power flow, a coupled inductor and the other voltage source are added into the input side. It keeps the advantage of push-pull M2DC and has additional degree of freedom. And using of coupled inductors avoid the $\mathrm{AC}$ current flowing into $\mathrm{DC}$ side. So that the topology structure bring higher flexibility, efficiency and stability for the DC-DC converter. As the result, the waveforms of simulation shows that DISO push-pull M2DC can be used in some way. It should be researched and improved in the future.

\section{Acknowledgements}

This work is supported by the Key Project of NSFC (No. 61533012), the Shanghai Natural Science Foundation (14ZR1421800), the State Key Laboratory of Synthetical Automation for Process Industries.

\section{References}

[1] H. Kakigano, Y. Miura, T. Ise, Low-voltage bipolar-type DC microgrid for super high quality distribution, IEEE Transactions on Power Electronics. 25 (2010) 3066-3075.

[2] S.P. Engel, M. Stieneker, N. Soltau, et al, Comparison of the modular multilevel dc converter and the dual-active bridge converter for power conversion in HVDC and MVDC grids, IEEE Transactions on Power Electronics. 30 (2015) 124-137.

[3] J.A. Ferreira, The multilevel modular dc converter, IEEE Transactions on Power Electronics. 28 (2013) 4460-4465.

[4] S. Kenzelmann, A. Rufer, D. Dujic, et al, A versatile DC/DC converter based on Modular Multilevel Converter for energy collection and distribution, IET Conference on Renewable Power Generation (RPG 2011). (2011) 1-6. 